\title{
Kawasaki disease shock syndrome: three cases
}

\author{
*Manoji Gitanjali Sathiadas ${ }^{1}$, Nirupa Umashankar ${ }^{1}$, Reginold Ragunathan $^{2}$, Kasthuri Thangarajah ${ }^{2}$
}

Sri Lanka Journal of Child Health, 2017; 46(4): 381-383

DOI: http://dx.doi.org/10.4038/sljch.v46i4.8391

(Key words: Kawasaki disease shock syndrome, 2-D echocardiography, shock, coronary arteries)

\section{Introduction}

Kawasaki disease (KD) is a disease of unknown aetiology affecting medium sized blood vessels ${ }^{1}$. It is the commonest type of acquired heart disease in children and $15-25 \%$ of patients have coronary artery involvement $^{2}$. Kawasaki disease shock syndrome (KDSS) is a rare haemodynamically unstable phenomenon in the acute stages of $\mathrm{KD}$ which was first described by Kenagaye et al in $2009^{1}$. Possible causes for the haemodynamic instability are vasculitis with capillary leak, myocardial dysfunction and cytokine dysregulation ${ }^{1}$. KDSS may be misdiagnosed as it mimics other causes of shock ${ }^{3}$. It is important to have a high index of suspicion for $\mathrm{KD}$ in any child with prolonged fever of unknown origin and to refer to a paediatric facility promptly, as timely treatment reduces coronary artery damage ${ }^{3}$. We report three cases of KDSS seen over a period of one year needing care in the high dependency and intensive care units.

\section{Case 1}

A 10-year-old girl presented with a history of fever for 2 days and an episode of vomiting. On examination, she was febrile with tachycardia and signs of circulatory collapse (hypotension with poor peripheral perfusion). There were no changes in the mucous membranes, no rashes, no cervical lymphadenopathy and no inflamed BCG scar. Differential diagnoses considered were dengue, leptospirosis, sepsis, myocarditis and KD. The white blood cell (WBC) count was $5,600 / \mathrm{cu} \mathrm{mm}$, the platelet count $42,000 / \mathrm{cu} \mathrm{mm}$, the C-reactive protein (CRP) $177 \mathrm{mg} / \mathrm{dl}$ and the erythrocyte sedimentation rate (ESR) $70 \mathrm{~mm}$ in the first hour.

${ }_{1}^{1}$ University of Jaffna, Sri Lanka, ${ }^{2}$ Teaching Hospital, Jaffna, Sri Lanka

*Correspondence: docsathiadas@hotmail.com

(Received on 17 August 2016: Accepted after revision on 23 September 2016)

The authors declare that there are no conflicts of interest

Personal funding was used for the project.

Open Access Article published under the Creative

Commons Attribution CC-BY (cC) (i)
Her liver function tests were abnormal with aspartate aminotransferase (AST) 2066U/L and alanine aminotransferase (ALT) 1427U/L. Blood culture was negative. The electrocardiogram (ECG) showed sinus tachycardia with no ischaemic changes, and serum troponin I was within normal range $(0.019 \mathrm{ng} / \mathrm{ml})$. Dengue antibody was negative. She was treated with intravenous (IV) cefotaxime, normal saline boluses and maintenance IV fluid. The 2D-echocardiogram, done on day 3 of admission, showed uniformly dilated right and left coronary arteries, with an ejection fraction of $55 \%$. (Figure 1 ).

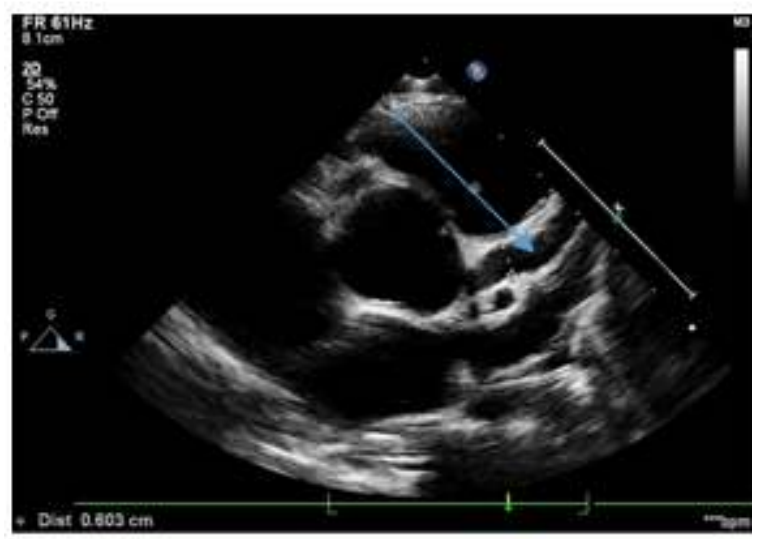

Figure 1: 2D-echocardiogram showing dilated left anterior descending coronary artery

The ultrasound scan (USS) of the abdomen was normal. Blood picture excluded microangiopathic haemolytic anaemia and disseminated intravascular coagulation (DIC). She was treated with IV human immunoglobulin $2 \mathrm{~g} / \mathrm{kg}$. Anti-platelet dose of aspirin was commenced when the platelet count rose to more than $400,000 / \mathrm{cu} \mathrm{mm}$. She developed peeling of skin during the 2 nd week of illness. Coronary angiogram also showed dilated coronaries. She is currently on regular follow up with 2D-echocardiograms.

\section{Case 2}

An 11-year-old boy, with a history of fever and throat pain of 4 days duration, presented in shock with unrecordable blood pressure. $\mathrm{He}$ had cervical lymphadenopathy, redness and swelling of lips and peeling of skin in the hands and feet. The WBC count was $10,000 / \mathrm{cu} \mathrm{mm}$ and the platelet count $14,000 / \mathrm{cu}$ 
mm. The CRP and ESR were high. Troponin I was normal and dengue antibody was negative. There was no ischaemic change in ECG. 2D-echocardiogram showed dilated left anterior descending and right coronary artery, a thin rim of pericardial effusion and an ejection fraction of $50 \%$. He was managed with IV human immunoglobulin $2 \mathrm{~g} / \mathrm{kg}$ and anti-platelet doses of aspirin and clopidogrel, when the risk of bleeding was excluded. Follow up echocardiogram and coronary angiogram showed progressive dilatation of left anterior descending artery. The 6 month followup also showed persistence of the dilated coronaries.

\section{Case 3}

A 4 year-old boy was admitted with a 6 day history of high grade fever and generalized erythematous rash. He also had bilateral non purulent conjunctivitis and right submandibular lymphadenopathy. He was haemodynamically stable on admission but went into circulatory collapse on the second day of admission. There was a $4 \mathrm{~cm}$ tender hepatomegaly. The haemoglobin level was $10.6 \mathrm{~g} / \mathrm{dl}$, the WBC count $10,390 / \mathrm{cu} \mathrm{mm}$, the platelet count $65,000 / \mathrm{cu} \mathrm{mm}$, packed cell volume (PCV) $29.4 \%$, ESR 70mm in first hour, CRP $160.5 \mathrm{mg} / \mathrm{L}$, sodium $134 \mathrm{mEq} / 1$, potassium $3.9 \mathrm{mEq} / 1$, blood urea $8.9 \mathrm{mmol} / 1$, aspartate transaminase (AST) 55U/L and gamma glutamyl transferase (GGT) 80U/1. The dengue antigen and antibody were negative and troponin I was $0.11 \mu \mathrm{g} / \mathrm{l}$. Blood picture showed a reactive white cell picture and mild thrombocytopenia. Ultrasound scan of the abdomen and neck revealed hepatosplenomegaly and mild ascites and lymphadenitis respectively. Echocardiography revealed mildly reduced left ventricular function, thin rim of pericardial effusion, uniformly dilated right $(4 \mathrm{~mm})$ and left $(4 \mathrm{~mm})$ coronary arteries and no aneurysms of the coronaries (Figure 2).

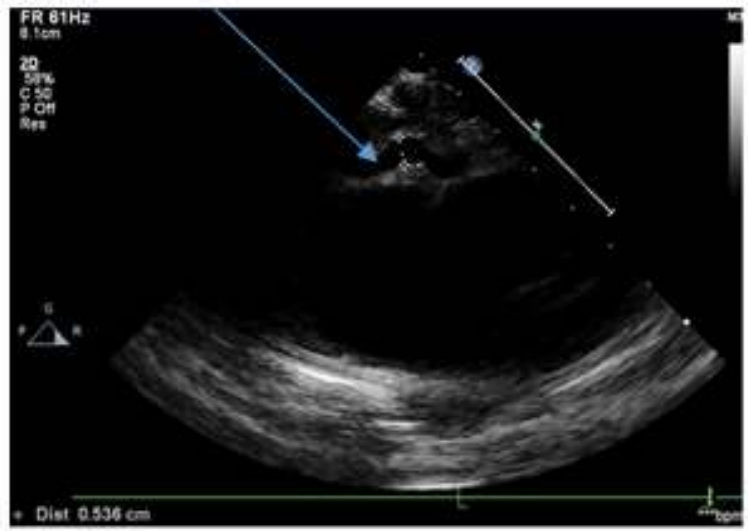

Figure 2: 2D-echocardiogram showing dilated right coronary artery
He was managed in the intensive care unit with inotropic support and IV immunoglobulin. Antiplatelet treatment was started when the platelet count rose to $500,000 / \mathrm{cu} \mathrm{mm}$. As the family lives abroad, details of follow up are not known.

\section{Discussion}

Literature shows the disease is predominant in the 3 3.5 year age group but the cases reported here are older children ${ }^{3}$. Males have shown a preponderance and the clinical feature of erythematous rash was prominent $^{2}$. KDSS can also present without any of the typical features of $\mathrm{KD}^{3}$. Detecting and suspecting KDSS is difficult as it clearly mimics dengue shock syndrome, toxic shock and septic shock ${ }^{2}$. The presence of high inflammatory markers with deranged liver function test can mislead the clinician. Performing 2D echocardiography early in the disease and having a suspicion of KDSS can help the clinician to pick this disease early ${ }^{3}$. The persistent nature of tachycardia prompted the clinicians to perform 2D Echocardiography in all three cases we report.

One published research article showed that compared with patients with $\mathrm{KD}$, patients with KDSS had a higher CRP concentration, and lower haemoglobin concentrations and platelet counts ${ }^{3}$. Evidence of consumptive coagulopathy was common in $\mathrm{KDSS}^{4}$. Patients with KDSS more often had impaired left ventricular systolic function, mitral regurgitation and coronary artery abnormalities ${ }^{2}$. All three patients had high inflammatory markers, and low platelet levels with impaired left ventricular systolic function and coronary artery abnormalities.

The coronary artery abnormalities were seen during acute, convalescent and chronic phases of the disease more prominently in KDSS when compared to $\mathrm{KD}^{5}$. All the cases reported here also show the persistent nature of the coronary artery abnormalities even after timely diagnosis and timely administration of IVIG therapy. It is also shown that IVIG can be resistant in $\mathrm{KDSS}^{4}$. This may be the reason for the persistence of the coronary artery disease. More prominent inflammatory markers are found in these patients. We suppose more severe inflammation may make them more likely to be IVIG resistant.

It is recommended that refractory disease is first treated with a second dose of IVIG $2 \mathrm{~g} / \mathrm{kg}$, though there are a number of other therapeutic options, including IV corticosteroid pulse therapy, anti-TNFalpha antibodies, and cytotoxic agents ${ }^{2,5}$. In the presence of coronary artery disease, the children 
should be on aspirin and clopidogrel to prevent thrombotic events.

Patients with KDSS may have an uneven clinical course and may be misdiagnosed when they are first examined. They may have more prominent inflammatory markers and result in shock and hypotension, which require critical care support at an early stage. They have a greater risk of coronary artery abnormalities, which may become coronary artery disease. These patients frequently fail to fulfil the full spectra of KD in the early phase, so delay in IVIG treatment may occur. They are also likely to be IVIG resistant because of more severe inflammation. Early recognition of KDSS and provision of adequate therapy are very important.

\section{References}

1. Kanegaye JT, Wilder MS, Molkara D, Frazer JR, Pancheri J, Tremoulet AH, et al. Recognition of a Kawasaki Disease Shock Syndrome. Pediatrics 2009; 123(5): e783-9 https://doi.org/10.1542/peds.2008-1871 PMid: 19403470 PMCid: PMC2848476
2. Jamieson N, Singh-Grewal D. Kawasaki Disease: A Clinician's Update. International Journal of Pediatrics 2013; 2013; ArticleID645391.

3. Chen P-S, Chi H, Huang F-Y, Peng C-C, Chen M-R, Chiu N-C. Clinical manifestations of Kawasaki disease shock syndrome: A case-control study. Journal of Microbiology, Immunology and Infection 2015; 48(1): 43-50. https://doi.org/10.1016/j.jmii.2013.06.005

PMid: 23927822

4. Eleftheriou D, Levin M, Shingadia D, Tulloh R, Klein NJ, Brogan PA. Management of Kawasaki disease, Archives of Disease in Childhood 2014; 99:74-83. https://doi.org/10.1136/archdischild-2012302841

PMid: 24162006 PMCid: PMC3888612

5. Newburger JW, Takahashi M, Gerber MA, et al. Diagnosis, treatment, and long term management of Kawasaki disease. Circulation 2004; 110:2747-71. https://doi.org/10.1161/01.CIR.0000145143. 19711.78

PMid: 15505111 